\title{
The Lojasiewicz Exponent at Infinity of Non-negative and Non-degenerate Polynomials
}

\author{
Grzegorz Oleksik $^{1}$ • Adam Różycki ${ }^{1}$
}

Received: 16 June 2017 / Accepted: 21 February 2018 / Published online: 2 March 2018

(C) The Author(s) 2018. This article is an open access publication

\begin{abstract}
Let $f$ be a real polynomial, non-negative at infinity with non-compact zeroset. Suppose that $f$ is non-degenerate in the Kushnirenko sense at infinity. In this paper we give a formula for the Łojasiewicz exponent at infinity of $f$ and a formula for the exponent of growth of $f$ in terms of its Newton polyhedron.
\end{abstract}

Keywords Łojasiewicz exponent · Newton polyhedron · Kushnirenko nondegeneracy

Mathematics Subject Classification 14P10

\section{Introduction}

Let $f: \mathbb{R}^{n} \rightarrow \mathbb{R}$ be a real polynomial, $f(0)=0$ and $K \subset \mathbb{R}^{n}$ be a compact set. The well known classical inequality (see Łojasiewicz 1959) say that there exist positive constants $C, \alpha$ such that

$$
|f(x)| \geq C \cdot \operatorname{dist}\left(x, f^{-1}(0)\right)^{\alpha},
$$

for all $x \in K$.

If the set $K$ is non-compact, it may happen that such $C, \alpha$ do not exist. One may check that it is impossible for polynomials

Adam Różycki

rozycki@math.uni.lodz.pl

Grzegorz Oleksik

oleksig@math.uni.lodz.pl

1 Faculty of Mathematics and Computer Science, University of Lodz, S. Banacha 22, 90-238 Lodz, Poland 


$$
f(x, y, z)=x^{2}\left(y^{2}+z^{4}\right) \text { and } f(x, y)=x(y-1)\left[y^{2}+(x y-1)^{2}\right] .
$$

For this reason, some authors modify inequality (1) or its domain.

Hörmander (1958) considered a global version of inequality (1). Precisely, he proved the following

$$
|f(x)| \cdot(1+|x|)^{\beta} \geq C \cdot \operatorname{dist}\left(x, f^{-1}(0)\right)^{\alpha},
$$

for all $x \in \mathbb{R}^{n}$ and some positive constants $C, \alpha, \beta$.

In some additional assumptions another global version of inequality (1) was given in Đinh (2014) i.e.

$$
|f(x)|^{\alpha}+|f(x)|^{\beta} \geq C \cdot \operatorname{dist}\left(x, f^{-1}(0)\right), x \in \mathbb{R}^{n},
$$

for some positive constants $C, \alpha, \beta$.

In turn, Hà and Duc (2010) Ha and Nguyen modified the zero set of polynomial $f: \mathbb{R}^{2} \rightarrow \mathbb{R}$ in inequality (1):

$$
|f(x)| \geq C \cdot \operatorname{dist}\left(x, f^{-1}(0)^{\mathbb{R}}\right)^{\alpha},
$$

in some neighborhood at infinity, where $f^{-1}(0)^{\mathbb{R}}$ denotes real approximation at infinity of $\left\{x \in \mathbb{C}^{2}: f(x)=0\right\}$.

Another modification concerned both a zero set and a domain. Indeed, in Đinh (2013) Kurdyka and Le Gal established

$$
|f(x)| \geq C \cdot \operatorname{dist}(x, Z)^{\alpha}, x \in f^{-1}(-\delta, \delta)
$$

for some positive constants $\delta, C, \alpha$, where $Z=\left\{x \in \mathbb{R}^{n}: f(x) \cdot \frac{\partial f}{\partial x_{1}}(x)=0\right\}$, and $f$ is a monic polynomial with respect to $x_{1}$. In this case constants $C, \alpha$ can be computed explicitly (see Hà et al. 2015).

If the set $f^{-1}(0)$ is compact, then

$$
\operatorname{dist}\left(x, f^{-1}(0)\right) \approx|x| .
$$

In this case for real polynomial $f: \mathbb{R}^{n} \rightarrow \mathbb{R}$, Gwoździewicz (1998) proved the following

$$
|f(x)| \geq C \cdot|x|^{d-(d-1)^{n}},|x|>R,
$$

where $d=\operatorname{deg} f>2$ and $C, R>0$.

Kollár (1988) gave similar result for complex polynomial mappings $F: \mathbb{C}^{n} \rightarrow \mathbb{C}^{n}$, $\# F^{-1}(0)<\infty$ i.e.

$$
|F(x)| \geq C \cdot|x|^{d-d^{n}},|x|>R,
$$


where $d=\operatorname{deg} F$ and $C, R>0$.

In the paper we assume that $f^{-1}(0)$ is a non-compact set. We keep the form of inequality (1), but we restrict the domain. Namely, we examine behavior of $f$ :

(i) in the neighborhood of the level set $f^{-1}(0)$ at infinity i.e. in the set

$$
\left\{x \in \mathbb{R}^{n}: \operatorname{dist}\left(x, f^{-1}(0)\right)<\varepsilon,|x|>R\right\},
$$

(ii) or in the set

$$
\left\{x \in \mathbb{R}^{n}: \operatorname{dist}\left(x, f^{-1}(0)\right)>R\right\} .
$$

The lack of the distinction of these cases could lead to a situation that an exponent $\alpha$ in inequality (1) does not exist in neighborhood at infinity. See for example polynomials (2). In the case (i) and (ii) we give the following definitions.

Let $f: \mathbb{R}^{n} \rightarrow \mathbb{R}$ be a polynomial such that $f^{-1}(0)$ is a non-compact set. We define the Łojasiewicz exponent of $f$ at infinity as the infimum of the exponents $l \in \mathbb{R}_{+}$such that

$$
|f(x)| \geq C \cdot \operatorname{dist}\left(x, f^{-1}(0)\right)^{l} \text { for all } x \text { such that } \operatorname{dist}\left(x, f^{-1}(0)\right)<\varepsilon,
$$

in some neighborhood of infinity for some $\varepsilon>0$ and $C>0$. We denote it by $\mathcal{L}_{\infty}(f)$. In cases where such $l$ does not exist, we put

$$
\mathcal{L}_{\infty}(f)=+\infty
$$

In Đinh (2012) authors proved that there are no sequences of the first type if and only if there exist $C, \delta, \alpha>0$ such that

$$
|f(x)|^{\alpha} \geq C \cdot \operatorname{dist}\left(x, f^{-1}(0)\right) \text { for all } x \in f^{-1}([-\delta, \delta])
$$

The sequence $\left(x_{k}\right)_{k=1}^{\infty} \subset \mathbb{R}^{n}$ is of the first type if $f\left(x_{k}\right) \rightarrow 0$ and $\operatorname{dist}\left(x, f^{-1}(0)\right) \nrightarrow 0$.

It is easy to observe that if the last inequality is true for some positive $C, \delta, \alpha$, then there exist $C, \varepsilon, l>0$ such that inequality (3) is true. Hence if there are no sequences of the first type, then $\mathcal{L}_{\infty}(f)$ exists. However, in some cases $\mathcal{L}_{\infty}(f)$ exists but there is a sequence of the first type. For example $f(x, y)=x(y-1)\left[y^{2}+(x y-1)^{2}\right]$.

In the paper we give an effective formula for the Łojasiewicz exponent at infinity in the class of non-negative and non-degenerate polynomials in terms of the Newton polyhedron (see Sect. 2). This result is a counterpart at infinity of the local result of the paper Bùi and Pham (2014).

\section{Preliminaries}

We denote by $\mathbb{R}_{+}=\{x \in \mathbb{R}: x \geq 0\}$ and $\mathbb{Z}_{+}=\mathbb{Z} \cap \mathbb{R}_{+}$. For $x=\left(x_{1}, \ldots, x_{n}\right) \in \mathbb{R}^{n}$ and $\alpha=\left(\alpha_{1}, \ldots, \alpha_{n}\right) \in \mathbb{Z}_{+}^{n}$, we denote by $x^{\alpha}$ the monomial $x_{1}^{\alpha_{1}} \ldots x_{n}^{\alpha_{n}}$ and put $|\alpha|=\alpha_{1}+\cdots+\alpha_{n}$, and $|x|=\max _{i=1}^{n}\left|x_{i}\right|$. 
Let $f(x)=\sum_{\alpha \in \mathbb{Z}_{+}^{n}} c_{\alpha} x^{\alpha}$. Let us define the set $\operatorname{supp}(f)=\left\{\alpha \in \mathbb{Z}_{+}^{n}: c_{\alpha} \neq 0\right\}$ and call it the support of $f$. Define the set $\Gamma(f)=\operatorname{conv}\{\operatorname{supp}(f)\} \subset \mathbb{R}_{+}^{n}$ and call it the Newton polyhedron at infinity of $f$.

Let $q \in \mathbb{R}^{n} \backslash\{0\}$. Define

$$
\begin{aligned}
d(q, \Gamma(f)) & =\min \{\langle q, \alpha\rangle: \alpha \in \Gamma(f)\} \\
\Delta(q, \Gamma(f)) & =\{\alpha \in \Gamma(f):\langle q, \alpha\rangle=d(q, \Gamma(f))\},
\end{aligned}
$$

where $\langle\cdot, \cdot\rangle$ denotes the standard inner product in $\mathbb{R}^{n} \times \mathbb{R}^{n}$. We say that $\Delta \subset \Gamma(f)$ is a face of $\Gamma(f)$, if there exists $q \in \mathbb{R}^{n} \backslash\{0\}$ such that $\Delta=\Delta(q, \Gamma(f))$. By a dimension of a face $\Delta$ we mean the minimum of the dimensions of the affine subspace containing $\Delta$. By a vertice of $\Gamma(f)$ we mean the 0 -dimensional faces of $\Gamma(f)$. We define Newton boundary at infinity of $f$ as the set of faces $\Delta \subset \Gamma(f)$ such that: if $q$ is defining a vector for $\Delta$ then $q_{i}<0$ for some $i \in\{1, \ldots, n\}$ and we denote it by $\Gamma_{\infty}(f)$. Denote by $\Gamma_{\infty}^{k}(f)$ the set of $k$-dimensional faces of $\Gamma_{\infty}(f), k=0, \ldots, n-1$. For $\Delta \in \Gamma_{\infty}(f)$ we define the polynomial

$$
f_{\Delta}=\sum_{\alpha \in \Delta} c_{\alpha} x^{\alpha} .
$$

and call it the principal part of $f$ at infinity with respect to face $\Delta$.

We say that $f$ is Kushnirenko non-degenerate at infinity on the face $\Delta \in \Gamma_{\infty}(f)$ if the system of equations

$$
\frac{\partial f_{\Delta}}{\partial x_{1}}(x)=\cdots=\frac{\partial f_{\Delta}}{\partial x_{n}}(x)=0
$$

has no solution in $(\mathbb{R} \backslash\{0\})^{n} \backslash K$, where $K \subset \mathbb{R}^{n}$ is a compact set. We say that $f$ is Kushnirenko non-degenerate at infinity (shortly non-degenerate) if $f$ is Kushnirenko non-degenerate at infinity on each face $\Delta \in \Gamma_{\infty}(f)$.

We say that $f$ is non-negative at infinity (shortly non-negative) if there exists a compact set $K \subset \mathbb{R}^{n}$ such that $f(x) \geq 0$ for $x \in \mathbb{R}^{n} \backslash K$.

One of the main tool which we use in the paper is the following

Lemma 1 (Curve Selection Lemma at infinity, Đinh (2014), Lemma 1) Let $A \subset \mathbb{R}^{n}$ be a semi-algebraic set, and let $F:=\left(f_{1}, \ldots, f_{p}\right): \mathbb{R}^{n} \rightarrow \mathbb{R}^{p}$ be a semi-algebraic map. Assume that there exists a sequence $x^{k} \in A$ such that $\lim _{k \rightarrow \infty}\left|x^{k}\right|=\infty$ and $\lim _{k \rightarrow \infty} F\left(x^{k}\right)=y \in(\overline{\mathbb{R}})^{p}$, where $\overline{\mathbb{R}}:=\mathbb{R} \cup\{ \pm \infty\}$. Then there exists an analytic curve $\varphi:(0, \epsilon) \rightarrow$ A of the form

$$
\varphi(t)=a^{0} t^{q}+a^{1} t^{q+1}+\ldots
$$

such that $a^{0} \in \mathbb{R}^{n} \backslash\{0\}, q<0, q \in \mathbb{Z}$, and $\lim _{t \rightarrow 0} F(\varphi(t))=y$.

Let $A \subset \mathbb{N}^{n}$ be a finite set. Put

$$
N_{A}(x)=\max _{\alpha \in A}\left|x^{\alpha}\right| .
$$


Let $V$ be the set of vertices of $\Gamma(f)$. Denote

$$
N_{\Gamma}=N_{V}
$$

We recall two simple lemmas which will be used in the rest of the paper.

Lemma 2 (Đinh (2014), Lemma 11) There exist some subset $J_{1}, \ldots, J_{s}$ of $\{1, \ldots, n\}$, with $J_{i} \nsubseteq J_{j}$ for $i \neq j$, such that

$$
N_{\Gamma}^{-1}(0)=\bigcup_{k=1}^{s} Z_{k}
$$

where $Z_{k}:=\left\{x \in \mathbb{R}^{n}: x_{j}=0, j \in J_{k}\right\}$.

For a given subset $J \subset\{1, \ldots, n\}$ we define

$$
\mathbb{R}^{J}:=\left\{\alpha=\left(\alpha_{1}, \ldots, \alpha_{n}\right) \in \mathbb{R}^{n}: \alpha_{j}=0 \text { for } j \notin J\right\} .
$$

Lemma 3 (Đinh (2014), Lemma 12) Let $J_{1}, \ldots, J_{s}$ be as in Lemma 2. For every $\left(j_{1}, \ldots, j_{s}\right) \in J_{1} \times \cdots \times J_{s}$, we have $V \cap \mathbb{R}^{J} \neq \emptyset$, where $J=\left\{j_{1}, \ldots, j_{s}\right\}$.

\section{The Main Theorem}

Let $J_{1}, \ldots, J_{s}$ be as in Lemma 2 and let

$$
\mathcal{P}=\left\{I \subset\{1, \ldots, n\}: I \neq \emptyset \wedge I \cap J_{k}=\emptyset \text { for some } k \in\{1, \ldots, s\}\right\} .
$$

Observe that $\mathcal{P} \neq \varnothing$ i.e. $J_{k_{0}} \neq\{1, \ldots, n\}$ for some $k_{0} \in\{1, \ldots, s\}$. Indeed, suppose to the contrary that $J_{k}=\{1, \ldots, n\}$ for any $k \in\{1, \ldots, s\}$. If $s>1$, then by Lemma 2 it is not possible. Therefore $s=1$. Hence $J_{1}=\{1, \ldots, n\}$ and $N_{\Gamma}^{-1}(0)=\{0\}$. By Lemma 10

$$
f^{-1}(0) \cap\left(\mathbb{R}^{n} \backslash K\right)=N_{\Gamma}^{-1}(0) \cap\left(\mathbb{R}^{n} \backslash K\right)=\emptyset,
$$

for some compact set $K$. This gives a contradiction to the assumption that the set $f^{-1}(0)$ is not compact.

Let us fix $I \in \mathcal{P}$. We define $\varphi^{I}(x)=\left(\varphi_{1}^{I}(x), \ldots, \varphi_{n}^{I}(x)\right)$, where

$$
\varphi_{i}^{I}(x)=\left\{\begin{array}{c}
1 \text { for } i \in I \\
x_{i} \text { for } i \notin I
\end{array}\right.
$$

for $i=1, \ldots, n$ and define $N_{\Gamma}^{I}=N_{\Gamma} \circ \varphi^{I}$.

Observe that

$$
\left(N_{\Gamma}^{I}\right)^{-1}(0)=\bigcup_{k=1}^{s(I)} Z_{k}^{I}=\bigcup_{k=1}^{s(I)}\left\{x \in \mathbb{R}^{I^{\prime}}: x_{j}=0, j \in J_{k}^{I}\right\}, \quad J_{l}^{I} \nsubseteq J_{m}^{I}, l \neq m,
$$


where $I^{\prime}=\{1, \ldots, n\} \backslash I$. Put

$$
\alpha_{\max }^{I}=\max \left\{\alpha_{J}^{\min }: J \in J_{1}^{I} \times \cdots \times J_{s(I)}^{I}\right\}
$$

where

$$
\alpha_{J}^{\min }:=\min \left\{|\alpha|: \alpha \in V^{I^{\prime}} \cap \mathbb{R}^{J}\right\}
$$

and $V^{I^{\prime}}$ denotes the projection of the set $V$ onto $\mathbb{R}^{I^{\prime}}$. Observe that $N_{\Gamma}^{I}=N_{V^{I^{\prime}}}$.

Now, we give the main result of the paper.

Theorem 4 Let $f: \mathbb{R}^{n} \rightarrow \mathbb{R}, f(0)=0$, be a non-negative and non-degenerate polynomial. Then

$$
\mathcal{L}_{\infty}(f)=\max \left\{\alpha_{\max }^{I}: I \in \mathcal{P}\right\}
$$

Remark 5 One can check that the assertions of the above theorems are also true if we assume Mikhailov-Gindikin non-degeneracy (see Đinh (2014), Section 5).

To illustrate the above theorems we give the following

Example 6 Let $f(x, y, z)=x^{8}\left(y^{4}+z^{6}\right)$. It is easy to see that $f$ is non-degenerate and non-negative. We have $V=\{(8,4,0),(8,0,6)\}$ and

$$
N_{\Gamma}(x, y, z)=\max \left\{x^{8} y^{4}, x^{8} z^{6}\right\}, \quad\left(N_{\Gamma}\right)^{-1}(0)=\{x=0\} \cup\{y=z=0\} .
$$

Hence $J_{1}=\{1\}, J_{2}=\{2,3\}$.

We calculate $\mathcal{L}_{\infty}(f)$. We have $\mathcal{P}=\{\{1\},\{2\},\{3\},\{2,3\}\}$. For $I=\{1\}$ we obtain $I^{\prime}=\{2,3\}$ and

$$
N_{\Gamma}^{I}(y, z)=\max \left\{y^{4}, z^{6}\right\}, \quad\left(N_{\Gamma}^{I}\right)^{-1}(0)=\{(0,0)\}, \quad J_{1}^{I}=\{2,3\} .
$$

Hence $J=\{2\}$ or $J=\{3\}$ and

$$
\alpha_{2}^{\min }=\min \{|\alpha|: \alpha \in\{4,0\}\}=4, \quad \alpha_{3}^{\min }=\min \{|\alpha|: \alpha \in\{0,6\}\}=6 .
$$

Therefore

$$
\alpha_{\max }^{\{1\}}=\max \{4,6\}=6 .
$$

Similarly we calculate

$$
\alpha_{\max }^{\{2\}}=\alpha_{\max }^{\{3\}}=\alpha_{\max }^{\{2,3\}}=8
$$

Finally we have

$$
\mathcal{L}_{\infty}(f)=\max \left\{\alpha_{\max }^{I}: I \in \mathcal{P}\right\}=\max \{6,8\}=8
$$




\section{Auxiliary Results}

The following lemmas will be used in the proof of Lemma 10. The proof of Lemma 7 is a simple transfer of its local counterpart [see Bùi and Pham (2014), Lemma 3.1]. We give it for a convenience of the reader.

Lemma 7 Suppose that $f$ is non-negative polynomial. Then for any face $\Delta \in \Gamma_{\infty}(f)$ we have $f_{\Delta}(x) \geq 0$ for $x \in(\mathbb{R} \backslash\{0\})^{n} \backslash K$, where $K$ is a compact set.

Proof Since $f$ is non-negative there exists a compact set $K$ such that $f(x) \geq 0$ for $x \in \mathbb{R}^{n} \backslash K$. Suppose to the contrary that there exists a face $\Delta \in \Gamma_{\infty}(f)$ and there exists a point $x^{0} \in(\mathbb{R} \backslash\{0\})^{n} \backslash K$ such that $f_{\Delta}\left(x^{0}\right)<0$. Let $J$ be the smallest subset of $\{1, \ldots, n\}$ such that $\Delta \subset \mathbb{R}^{J}$. Hence, there exists a non-zero vector $a \in \mathbb{R}^{n}$, with $a_{j}<0$ for some $j \in J$ and $a_{j}=0$ for $j \notin J$ such that

$$
\Delta=\left\{v \in \Gamma(f) \cap \mathbb{R}^{J}:\langle a, v\rangle=d(a, \Gamma(f))\right\} .
$$

Define monomial curve $\varphi:(0,1) \rightarrow \mathbb{R}^{n}, t \mapsto\left(\varphi_{1}(t), \ldots, \varphi_{n}(t)\right)$, by

$$
\varphi_{j}(t)=\left\{\begin{array}{cc}
x_{j}^{0} t^{a_{j}} & \text { for } j \in J, \\
0 & \text { for } j \notin J .
\end{array}\right.
$$

Put $d:=d(a, \Gamma(f))$. Now, we may write $f$ in the form:

$$
f(\varphi(t))=f_{\Delta}\left(x^{0}\right) t^{d}+\text { higher order terms in } t .
$$

Since $f_{\Delta}\left(x^{0}\right)<0$, we have

$$
f(\varphi(t))<0 \text { for all sufficiently small } t .
$$

This gives a contradiction.

However counterpart of equivalence Bùi and Pham (2014, Lemma 3.2) is not true at infinity. The simple implication is the only one that holds.

Lemma 8 If $f$ is non-negative and non-degenerate, then for any face $\Delta \in \Gamma_{\infty}(f)$ we have $f_{\Delta}>0$ on $(\mathbb{R} \backslash\{0\})^{n} \backslash K$.

Proof Using Lemma 7 we obtain $f_{\Delta}(x) \geq 0$ for all $x \in(\mathbb{R} \backslash\{0\})^{n} \backslash K$, where $K$ is a suitably chosen compact set. Suppose to the contrary that there exists a point $x^{0} \in(\mathbb{R} \backslash\{0\})^{n} \backslash K$ such that $f_{\Delta}\left(x^{0}\right)=0$. Therefore the function $f_{\Delta}$ attains a local minimum at the point $x^{0}$. Hence $\operatorname{grad} f_{\Delta}\left(x^{0}\right)=0$. This gives a contradiction to non-degeneracy of $f$. 
The following lemma will be also applied in the proof of the Lemma 10.

Lemma 9 Gindikin (1974, Lemma 1) Let $v \in \mathbb{R}_{+}^{n}, v \in \operatorname{conv}\left\{v^{1}, \ldots, v^{k}\right\}$. Then

$$
\left|x^{v}\right| \leq \sum_{j=1}^{k}\left|x^{v_{j}}\right|
$$

The next lemma plays a crucial role in the proof of the main theorem. Its proof is a substantially analogous to the proof of Lemma 3.3 of the paper Bùi and Pham (2014). However we prove the second inequality in (5) without assumption of non-degeneracy and non-negativity, using Lemma 9.

Lemma 10 If $f$ is non-negative and non-degenerate then there exist some positive constants $C_{1}$ and $C_{2}$ such that

$$
C_{1} N_{\Gamma}(x) \leq f(x) \leq C_{2} N_{\Gamma}(x), \text { for all } x \in \mathbb{R}^{n} \backslash K,
$$

for some compact set $K \subset \mathbb{R}^{n}$.

Proof We will prove the first inequality. Suppose to the contrary that there exists a sequence $\left\{x^{k}\right\} \subset \mathbb{R}^{n}$ with $\left|x^{k}\right|>k$ and such that

$$
f\left(x^{k}\right)<\frac{1}{k} N_{\Gamma}\left(x^{k}\right)
$$

for all $k$. By Lemma 1 , there exist an analytic curves $\varphi:(0, \epsilon) \rightarrow \mathbb{R}^{n}, t \mapsto$ $\left(\varphi_{1}(t), \ldots, \varphi_{n}(t)\right)$ and $\psi:(0, \epsilon) \rightarrow \mathbb{R}_{+}$such that

$$
|\varphi(t)| \rightarrow \infty, \quad|\psi(t)| \rightarrow 0 \quad \text { as } t \rightarrow 0^{+},
$$

and

$$
f(\varphi(t))<\psi(t) N_{\Gamma}(\varphi(t)) .
$$

Let $J=\left\{j: \varphi_{j} \not \equiv 0\right\} \subset\{1, \ldots, n\}$. For $j \in J$ we can expand coordinate function $\varphi_{j}$, say

$$
\varphi_{j}(t)=x_{j}^{0} t^{a_{j}}+\text { higher order terms in } t
$$

where $x_{j}^{0} \neq 0$ and $a_{j} \in \mathbb{N}$. From Condition (6), there exists $j \in J$ such that $a_{j}<0$. If $\Gamma(f) \cap \mathbb{R}^{J}=\emptyset$, then for any vertex $\alpha \in V$, there exists $j \notin J$ such that $\alpha_{j}>0$ $(V \subset \Gamma(f))$ and hence $\left(\varphi_{j}(t)\right)^{\alpha_{j}} \equiv 0$. Then $(\varphi(t))^{\alpha_{j}} \equiv 0$. Hence

$$
N_{\Gamma}(\varphi(t))=\max _{\alpha \in V}\left|\varphi(t)^{\alpha}\right| \equiv 0 .
$$

This gives a contradiction to (7). 
Therefore, $\Gamma(f) \cap \mathbb{R}^{J} \neq \emptyset$. Put

$$
\begin{aligned}
& d=\min \left\{\sum_{j \in J} a_{j} \alpha_{j}: \alpha \in \Gamma(f) \cap \mathbb{R}^{J}\right\}, \\
& \Delta=\left\{\alpha \in \Gamma(f) \cap \mathbb{R}^{J}: \sum_{j \in J} a_{j} \alpha_{j}=d\right\} .
\end{aligned}
$$

We can write

$$
f(\varphi(t))=f_{\Delta}\left(x^{0}\right) t^{d}+\text { higher order terms in } t,
$$

where $x^{0}=\left(x_{1}^{0}, \ldots, x_{n}^{0}\right)$ and $x_{j}^{0}=1$ for $j \notin J$. We will show that $f_{\Delta}\left(x^{0}\right)>0$. Indeed, since $f$ is non-negative and non-degenerate, it follows from Lemma 8 we have that $f_{\Delta}(x)>0$ for $x \in(\mathbb{R} \backslash\{0\})^{n} \backslash K$, where $K$ is a suitably chosen compact set. Therefore by quasi-homogeneity of $f_{\Delta}$ we have

$$
f_{\Delta}\left(x^{0}\right)=\frac{f_{\Delta}\left(\left(s^{a_{j}} x_{j}^{0}\right)_{j \in J}\right)}{s^{d}}>0,
$$

where $s$ is a positive number such that $s^{a_{j}} \cdot x_{j}^{0}$ is large enough for some $j \in J$. Hence

$$
f(\varphi(t)) \text { and } t^{d}
$$

are of the same order if $t \rightarrow 0^{+}$.

On the other hand, we have

$$
N_{\Gamma}(\varphi(t))=\max _{\alpha \in V}\left|\varphi(t)^{\alpha}\right|=\max _{\alpha \in \Delta}\left|\left(x^{0}\right)^{\alpha}\right| t^{d}+\text { higher order terms in } t .
$$

Hence and by (8) we have a contradiction to (7).

Now we prove the second inequality in (b). Let $|x| \geq R \geq 1$, where $R$ is sufficiently large. By Lemma 9 we have

$$
\begin{aligned}
f(x) & =\sum_{\nu \in \operatorname{supp} f} c_{\nu} x^{\nu} \leq \max _{\nu \in \operatorname{supp} f}\left|c_{\nu}\right| \cdot \sum_{\nu \in \operatorname{supp} f}\left|x^{\nu}\right| \leq \\
& \leq C_{2} \cdot \max _{\nu \in V}\left|x^{\nu}\right|=C_{2} \cdot N_{\Gamma}(x),
\end{aligned}
$$

where $C_{2}$ is a some positive constant.

Let $A \subset \mathbb{N}^{n}$ be a finite set. Put

$$
\mathcal{L}\left(N_{A}\right)=\inf \left\{l \in \mathbb{R}_{+}: \exists_{C>0}\left|N_{A}(x)\right| \geq C \cdot \operatorname{dist}\left(x, N_{A}^{-1}(0)\right)^{l}, \operatorname{dist}\left(x, N_{A}^{-1}(0)\right)<1\right\} .
$$

Now we give an effective formula to compute $\mathcal{L}\left(N_{A}\right)$. 


\section{Proposition 11 We have}

$$
\mathcal{L}\left(N_{A}\right)=\max \left\{\alpha_{J}^{\min }: J \in J_{1} \times \cdots \times J_{s}\right\}
$$

where

$$
\alpha_{J}^{\min }:=\min \left\{|\alpha|: \alpha \in A \cap \mathbb{R}^{J}\right\}
$$

Proof We first show that $\mathcal{L}\left(N_{A}\right) \leq \max \left\{\alpha_{J}^{\min }: J \in J_{1} \times \cdots \times J_{S}\right\}$. Let us fix an arbitrary $x \in \mathbb{R}^{n}$ such that

$$
\operatorname{dist}\left(x, N_{A}^{-1}(0)\right)=\delta<1 .
$$

It is easy to check that

$$
\operatorname{dist}\left(x, N_{A}^{-1}(0)\right)=\min _{k=1}^{s} \max _{j \in J_{k}}\left|x_{j}\right| \text {. }
$$

Hence

$$
\max _{j \in J_{k}}\left|x_{j}\right| \geq \delta \text { for any } k=1, \ldots, s .
$$

This means that for each $k=1, \ldots, s$ there exists $j_{k} \in J_{k}$ such that

$$
\left|x_{j_{k}}\right| \geq \delta
$$

Put $J=\left\{j_{1}, \ldots, j_{s}\right\}$. By Lemma 3 we have that $A \cap \mathbb{R}^{J} \neq \emptyset$. Let us choose $\alpha=$ $\left(\alpha_{1}, \ldots, \alpha_{s}\right) \in A \cap \mathbb{R}^{J}$ such that

$$
|\alpha|=\alpha_{J}^{\min }
$$

Hence

$$
\begin{aligned}
N_{A}(x) & =\max \left\{\left|x_{j_{1}}^{\alpha_{j}} \ldots x_{j_{s}}^{\alpha_{s}}\right|, \ldots\right\} \geq \delta^{\alpha_{j}} \ldots \delta^{\alpha_{s}} \\
& =\delta^{\alpha_{J}^{\min }} \geq \delta^{\max \left\{\alpha_{J}^{\min }: J \in J_{1} \times \cdots \times J_{s}\right\}} \\
& =\operatorname{dist}\left(x, N_{A}^{-1}(0)\right)
\end{aligned}
$$

This means that $\mathcal{L}\left(N_{A}\right) \leq \max \left\{\alpha_{J}^{\min }: J \in J_{1} \times \cdots \times J_{S}\right\}$.

Now, we show that $\mathcal{L}\left(N_{A}\right) \geq \max \left\{\alpha_{J}^{\min }: J \in J_{1} \times \cdots \times J_{S}\right\}$.

Let $\left(j_{1}, \ldots, j_{s}\right) \in J_{1} \times \cdots \times J_{s}$ be such that realized the above maximum and let $J \subset\{1, \ldots, n\}$ be the minimal set such that $j_{k} \in J, k=1, \ldots, s$. Put $A_{J}=$ 
$\mathbb{R}^{J} \cap A$. By Lemma 3 we have that $A_{J} \neq \emptyset$. Take the following parametrization $\varphi(t)=\left(\varphi_{1}(t), \ldots, \varphi_{n}(t)\right),|t|<1$, where

$$
\varphi_{i}(t)= \begin{cases}t & \text { for } i \in J \\ 0 & \text { for } i \notin J,\end{cases}
$$

for $i=1, \ldots, n$. We have

$$
N_{A}(\varphi(t))=\max _{v \in A_{J}}\left|\varphi(t)^{v}\right|=|t|^{\min \left\{|v|: v \in A_{J}\right\}}=|t|^{\alpha_{J}^{\min }}=\operatorname{dist}\left(\varphi(t), N_{A}^{-1}(0)\right)^{\alpha_{J}^{\min }}
$$

Hence $\mathcal{L}\left(N_{A}\right) \geq \alpha_{J}^{\min }$. This ends the proof.

One can observe that the above proof in comparison with the proof of (Bùi and Pham 2014, Proposition 3.1) is more elementary.

\section{Proof of the Main Theorem}

Now, we are ready to give the proof of the main result.

Proof of Theorem 4. Since $f$ is non-negative and non-degenerate polynomial, then by Lemma 10 there exist some positive constants $C_{1}$ and $C_{2}$ such that

$$
C_{1} N_{\Gamma}(x) \leq f(x) \leq C_{2} N_{\Gamma}(x)
$$

for all $x \in \mathbb{R}^{n} \backslash K$ and some compact set $K \subset \mathbb{R}^{n}$. Hence

$$
f^{-1}(0) \cap\left(\mathbb{R}^{n} \backslash K\right)=N_{\Gamma}^{-1}(0) \cap\left(\mathbb{R}^{n} \backslash K\right) .
$$

We will show that there exist some positive constants $D_{1}$ and $D_{2}$ such that

$$
D_{1} \operatorname{dist}\left(x, N_{\Gamma}^{-1}(0)\right) \leq \operatorname{dist}\left(x, f^{-1}(0)\right) \leq D_{2} \operatorname{dist}\left(x, N_{\Gamma}^{-1}(0)\right),
$$

for all $x \in \mathbb{R}^{n} \backslash K_{1}$ and some compact set $K_{1} \subset \mathbb{R}^{n}, K \subset K_{1}$. First, observe that

$$
\operatorname{dist}(x, K) \leq|x| \leq 2 \operatorname{dist}(x, K), \quad x \in \mathbb{R}^{n} \backslash K_{1},
$$

for some compact set $K_{1} \subset \mathbb{R}^{n}, K \subset K_{1}$. By (11), (12) and since $0 \in N_{\Gamma}^{-1}(0)$ we have

$$
\begin{aligned}
\operatorname{dist}\left(x, f^{-1}(0)\right) & =\min \left\{\operatorname{dist}\left(x, f^{-1}(0) \backslash K\right), \operatorname{dist}\left(x, f^{-1}(0) \cap K\right)\right\} \\
& \geq \min \left\{\operatorname{dist}\left(x, N_{\Gamma}^{-1}(0) \backslash K\right), \operatorname{dist}(x, K)\right\} \\
& \geq \min \left\{\operatorname{dist}\left(x, N_{\Gamma}^{-1}(0)\right), \frac{1}{2}|x|\right\}
\end{aligned}
$$




$$
\begin{aligned}
& \geq \min \left\{\operatorname{dist}\left(x, N_{\Gamma}^{-1}(0)\right), \frac{1}{2} \operatorname{dist}\left(x, N_{\Gamma}^{-1}(0)\right)\right\} \\
& =\frac{1}{2} \operatorname{dist}\left(x, N_{\Gamma}^{-1}(0)\right),
\end{aligned}
$$

for $x \in \mathbb{R}^{n} \backslash K_{1}$. Analogously, by (11), (12) and since $0 \in f^{-1}(0)$ we get

$$
\operatorname{dist}\left(x, N_{\Gamma}^{-1}(0)\right) \geq \frac{1}{2} \operatorname{dist}\left(x, f^{-1}(0)\right),
$$

for $x \in \mathbb{R}^{n} \backslash K_{1}$. Summing up we obtain

$$
\frac{1}{2} \operatorname{dist}\left(x, N_{\Gamma}^{-1}(0)\right) \leq \operatorname{dist}\left(x, f^{-1}(0)\right) \leq 2 \operatorname{dist}\left(x, N_{\Gamma}^{-1}(0)\right),
$$

for $x \in \mathbb{R}^{n} \backslash K_{1}$. By (10) and (13) it follows that

$$
\mathcal{L}_{\infty}\left(N_{\Gamma}\right)=\mathcal{L}_{\infty}(f) .
$$

By (14), it is enough to prove formula (4) for $N_{\Gamma}$. We first show that

$$
\mathcal{L}_{\infty}\left(N_{\Gamma}\right) \leq \max \left\{\mathcal{L}\left(N_{\Gamma}^{I}\right): I \in \mathcal{P}\right\}
$$

Let $x \in \mathbb{R}^{n} \backslash K$, where $K$ is the same as in Lemma 10 and $\operatorname{dist}\left(x, N_{\Gamma}^{-1}(0)\right)<\varepsilon<1$. It can be assumed that

$$
\left\{x \in \mathbb{R}^{n}:|x|<1\right\} \subset K .
$$

Let $I \neq \emptyset$ be such that

$$
\left|x_{i}\right| \geq 1, i \in I \text { and }\left|x_{i}\right|<1, i \notin I \text {. }
$$

It is easy to check that $I \in \mathcal{P}$. Since

$$
\left(N_{\Gamma}^{I}\right)^{-1}(0)=N_{\Gamma}^{-1}(0) \cap\left\{x \in \mathbb{R}^{n}: x_{i}=1 \text { for } i \in I\right\} \subset N_{\Gamma}^{-1}(0),
$$

we have

$$
\operatorname{dist}\left(x_{I},\left(N_{\Gamma}^{I}\right)^{-1}(0)\right) \geq \operatorname{dist}\left(x_{I}, N_{\Gamma}^{-1}(0)\right) .
$$

It is easy to check that

$$
\operatorname{dist}\left(x_{I}, N_{\Gamma}^{-1}(0)\right)=\operatorname{dist}\left(x, N_{\Gamma}^{-1}(0)\right) .
$$

By (16), (17) we obtain

$$
\left|N_{\Gamma}(x)\right| \geq\left|N_{\Gamma}^{I}\left(x_{I}\right)\right| \geq C_{I} \operatorname{dist}\left(x_{I},\left(N_{\Gamma}^{I}\right)^{-1}(0)\right)^{\mathcal{L}\left(N_{\Gamma}^{I}\right)}
$$




$$
\begin{aligned}
& \geq C_{I} \operatorname{dist}\left(x_{I}, N_{\Gamma}^{-1}(0)\right)^{\mathcal{L}\left(N_{\Gamma}^{I}\right)}=C_{I} \operatorname{dist}\left(x, N_{\Gamma}^{-1}(0)\right)^{\mathcal{L}\left(N_{\Gamma}^{I}\right)} \\
& \geq \min \left\{C_{I}: I \in \mathcal{P}\right\} \operatorname{dist}\left(x, N_{\Gamma}^{-1}(0)\right)^{\max \left\{\mathcal{L}\left(N_{\Gamma}^{I}\right): I \in \mathcal{P}\right\}} .
\end{aligned}
$$

This gives (15).

Now we show that

$$
\mathcal{L}_{\infty}\left(N_{\Gamma}\right) \geq \max \left\{\mathcal{L}\left(N_{\Gamma}^{I}\right): I \in \mathcal{P}\right\}
$$

First we choose $I \in \mathcal{P}$ such that realizes the above maximum. Take the parametrization $\varphi: \mathbb{R} \backslash\{0\} \rightarrow \mathbb{R}^{\{1, \ldots, n\} \backslash I}$ defined by formula (9) such that it realizes $\mathcal{L}\left(N_{\Gamma}^{I}\right)$. Let $\varepsilon>0$. Let $\left(\varphi_{\varepsilon}\right)_{i}: \mathbb{R} \backslash\{0\} \rightarrow \mathbb{R}^{n}$ be defined in the following way

$$
\left(\varphi_{\varepsilon}(t)\right)_{i}=\left\{\begin{array}{cc}
\varphi_{i}(t) & \text { for } i \notin I, \\
t^{-\varepsilon} & \text { for } i \in I,
\end{array}\right.
$$

Observe that

$$
\operatorname{dist}\left(\varphi_{\varepsilon}(t), N_{\Gamma}^{-1}(0)\right)=t .
$$

Indeed, let $K=\left\{k \in\{1, \ldots, s\}: J_{k} \cap I=\emptyset\right\}=\left\{k_{1}, \ldots, k_{r}\right\}$. We have

$$
\begin{aligned}
\operatorname{dist}\left(\varphi_{\varepsilon}(t), N_{\Gamma}^{-1}(0)\right) & =\min _{k=1}^{s} \max _{j \in J_{k}}\left|\varphi_{\varepsilon, j}(t)\right| \\
& =\min _{k=1}^{s}\left\{\max \left\{\max _{j \in J_{k} \cap I}\left|\varphi_{\varepsilon, j}(t)\right|, \max _{j \in J_{k} \backslash I}\left|\varphi_{\varepsilon, j}(t)\right|\right\}\right\} \\
& =\min _{k=1}^{s}\left\{\max \left\{\left|t^{-\varepsilon}\right|, \max _{j \in J_{k} \backslash I}\left|\varphi_{\varepsilon, j}(t)\right|\right\}\right\}=\min _{k \in K}\left\{\max _{j \in J_{k}}\left|\varphi_{j}(t)\right|\right\} .
\end{aligned}
$$

Now, it is enough to show that

$$
\min _{k \in K}\left\{\max _{j \in J_{k}}\left|\varphi_{j}(t)\right|\right\}=t .
$$

Observe that

$$
\begin{aligned}
\left(N_{\Gamma}^{I}\right)^{-1}(0) & =N_{\Gamma}^{-1}(0) \cap\left\{x \in \mathbb{R}^{n}: x_{i}=1, i \in I\right\} \\
& =\bigcup_{k=1}^{s} Z_{k} \cap\left\{x \in \mathbb{R}^{n}: x_{i}=1, i \in I\right\} \\
& =\bigcup_{k=1}^{s}\left\{x \in \mathbb{R}^{n}: x_{i}=0 \text { for } i \in J_{k}, x_{i}=1 \text { for } i \in I\right\}
\end{aligned}
$$




$$
\begin{aligned}
& =\bigcup_{k \in K}\left\{x \in \mathbb{R}^{n}: x_{i}=0 \text { for } i \in J_{k}, x_{i}=1 \text { for } i \in I\right\} \\
& =\bigcup_{l=1}^{r}\left\{x \in \mathbb{R}^{n}: x_{i}=0 \text { for } i \in J_{k_{l}}, x_{i}=1 \text { for } i \in I\right\} .
\end{aligned}
$$

Let $\left(j_{k_{1}}, \ldots, j_{k_{r}}\right) \in J_{k_{1}} \times \cdots \times J_{k_{r}}, J=\left\{j_{k_{1}}, \ldots, j_{k_{r}}\right\}$ be the same as in definition of $\varphi$. It is obvious that $J_{k_{l}} \cap J \neq \emptyset, l=1, \ldots, r$. Therefore

$$
\max _{j \in J_{k_{l}}}\left|\varphi_{j}(t)\right|=t, \quad l=1, \ldots, r .
$$

This gives (19).

Let $v_{I}$ be the system of these coordinates of $v$ which are in $I$ and $v_{I^{\prime}}$ - system of the remaining ones. We have

$$
\begin{aligned}
\left|\left(N_{\Gamma} \circ \varphi_{\varepsilon}\right)(t)\right| & =\max _{\nu \in V}\left|\varphi_{\varepsilon}(t)^{v}\right|=\max _{\nu \in V}\left\{\left|t^{-\varepsilon}\right|^{v_{I}} \cdot\left|\varphi_{I^{\prime}}(t)^{v_{I^{\prime}}}\right|\right\} \\
& \leq\left|t^{-\varepsilon}\right|^{\max _{v \in V}\left|v_{I}\right|} \cdot \max _{\nu \in V}\left|\varphi_{I^{\prime}}(t)^{v_{I^{\prime}}}\right|=\left|t^{-\varepsilon}\right|^{\max _{v \in V}\left|v_{I}\right|} \cdot N_{\Gamma}(\varphi(t)) \\
& =\left|t^{-\varepsilon}\right|^{\max _{v \in V}\left|v_{I}\right|} \cdot|t|^{\mathcal{L}\left(N_{\Gamma}^{I}\right)}=|t|^{\mathcal{L}\left(N_{\Gamma}^{I}\right)-\varepsilon \max _{v \in V}\left|v_{I}\right|} \\
& =\operatorname{dist}\left(\varphi_{\varepsilon}(t), N_{\Gamma}^{-1}(0)\right) \mathcal{L}\left(N_{\Gamma}^{I}\right)-\varepsilon \max _{v \in V}\left|v_{I}\right| .
\end{aligned}
$$

It can be assumed that $\varepsilon$ is such that $\mathcal{L}\left(N_{\Gamma}^{I}\right)-\varepsilon \max _{v \in V}\left|\nu_{I}\right|>0$. Hence

$$
\mathcal{L}_{\infty}\left(N_{\Gamma}\right) \geq \mathcal{L}\left(N_{\Gamma}^{I}\right)-\varepsilon \max _{\nu \in V}\left|\nu_{I}\right|
$$

By arbitrary choice of $\varepsilon$ and $I$ we obtain (18). Summing up we obtain

$$
\mathcal{L}_{\infty}\left(N_{\Gamma}\right)=\max \left\{\mathcal{L}\left(N_{\Gamma}^{I}\right): I \in \mathcal{P}\right\}
$$

By Proposition 11 we have $\mathcal{L}\left(N_{\Gamma}^{I}\right)=\alpha_{\max }^{I}$ and hence we get the formula (4) for $N_{\Gamma}$. This ends the proof.

\section{Formula of Exponent of Growth}

We also define the exponent of growth of $f$ at infinity as the supremum of the exponents $l \in \mathbb{R}_{+}$such that

$$
|f(x)| \geq C \cdot \operatorname{dist}\left(x, f^{-1}(0)\right)^{l} \text { for all } x \text { such that } \operatorname{dist}\left(x, f^{-1}(0)\right)>R,
$$

in some neighborhood of infinity for some $R>0$ and $C>0$. We denote it by $\mathcal{E}_{\infty}(f)$. In the case that such $l$ does not exist, we put

$$
\mathcal{E}_{\infty}(f)=-\infty
$$


The second result is a formula of exponent of growth of polynomial $f$ at infinity.

Theorem 12 Let $f: \mathbb{R}^{n} \rightarrow \mathbb{R}$ be a non-negative and non-degenerate polynomial. Then

$$
\mathcal{E}_{\infty}(f)=\min \left\{\alpha_{J}^{\max }: J \in J_{1} \times \cdots \times J_{s}\right\}
$$

where

$$
\alpha_{J}^{\max }:=\max \left\{|\alpha|: \alpha \in V \cap \mathbb{R}^{J}\right\}
$$

Proof By Lemma 10 we have $\mathcal{E}_{\infty}(f)=\mathcal{E}_{\infty}\left(N_{\Gamma}\right)$. Therefore it is enough to prove this formula for $N_{\Gamma}$. We first show that $\mathcal{E}_{\infty}\left(N_{\Gamma}\right) \geq \min \left\{\alpha_{J}^{\max }: J \in J_{1} \times \cdots \times J_{s}\right\}$. Let us fix arbitrary $x \in \mathbb{R}^{n} \backslash K$ such that

$$
\operatorname{dist}\left(x, N_{\Gamma}^{-1}(0)\right)=\delta>1
$$

Since

$$
\operatorname{dist}\left(x, N_{\Gamma}^{-1}(0)\right)=\min _{k=1}^{s} \max _{j \in J_{k}}\left|x_{j}\right|
$$

hence we get

$$
\max _{j \in J_{k}}\left|x_{j}\right| \geq \delta \text { for any } k=1, \ldots, s .
$$

This means that for each $k=1, \ldots, s$ there exists $j_{k} \in J_{k}$ such that

$$
\left|x_{j_{k}}\right| \geq \delta
$$

Put $J=\left\{j_{1}, \ldots, j_{s}\right\}$. By Lemma 3 we have $V \cap \mathbb{R}^{J} \neq \emptyset$. Let us choose $\alpha=$ $\left(\alpha_{1}, \ldots, \alpha_{s}\right) \in V \cap \mathbb{R}^{J}$ such that

$$
|\alpha|=\alpha_{J}^{\max }
$$

Hence

$$
\begin{aligned}
N_{\Gamma}(x) & =\max \left\{\left|x_{j_{1}}^{\alpha_{j}} \ldots x_{j_{s}}^{\alpha_{s}}\right|, \ldots\right\} \geq \delta^{\alpha_{j}} \ldots \delta^{\alpha_{s}} \\
& =\delta^{\alpha_{J}^{\max }} \geq \delta^{\min \left\{\alpha_{J}^{\max }: J \in J_{1} \times \cdots \times J_{s}\right\}} \\
& =\operatorname{dist}\left(x, N_{\Gamma}^{-1}(0)\right)^{\min \left\{\alpha_{J}^{\max }: J \in J_{1} \times \cdots \times J_{s}\right\}} .
\end{aligned}
$$

This means that $\mathcal{E}_{\infty}\left(N_{\Gamma}\right) \geq \min \left\{\alpha_{J}^{\max }: J \in J_{1} \times \cdots \times J_{s}\right\}$.

Now, we show that $\mathcal{E}_{\infty}\left(N_{\Gamma}\right) \leq \min \left\{\alpha_{J}^{\max }: J \in J_{1} \times \cdots \times J_{s}\right\}$. 
Let $\left(j_{1}, \ldots, j_{s}\right) \in J_{1} \times \cdots \times J_{s}$ and let $J \subset\{1, \ldots, n\}$ be the minimal set such that $j_{k} \in J, k=1, \ldots, s$. Put $V_{J}=\mathbb{R}^{J} \cap V$. By Lemma 3 we have that $V_{J} \neq \emptyset$. Take the following parametrization $\varphi(t)=\left(\varphi_{1}(t), \ldots, \varphi_{n}(t)\right),|t|>1$, where

$$
\varphi_{i}(t)=\left\{\begin{array}{lll}
t & \text { for } & i \in J \\
0 & \text { for } & i \notin J
\end{array}\right.
$$

for $i=1, \ldots, n$. We have

$$
N_{\Gamma}(\varphi(t))=\max _{\nu \in V_{J}}\left|\varphi(t)^{v}\right|=|t|^{\max \left\{|v|: v \in V_{J}\right\}}=|t|^{\alpha_{J}^{\max }}=\operatorname{dist}\left(\varphi(t), N_{\Gamma}^{-1}(0)\right)^{\alpha_{J}^{\max }} .
$$

Hence $\mathcal{E}_{\infty}\left(N_{\Gamma}\right) \leq \alpha_{J}^{\max }$ and by arbitrary choice of $\left(j_{1}, \ldots, j_{s}\right) \in J_{1} \times \cdots \times J_{s}$ we have

$$
\mathcal{E}_{\infty}\left(N_{\Gamma}\right) \leq \min \left\{\alpha_{J}^{\max }: J \in J_{1} \times \cdots \times J_{s}\right\} .
$$

This ends the proof.

Example 13 Let again $f(x, y, z)=x^{8}\left(y^{4}+z^{6}\right)$ and $V=\{(8,4,0),(8,0,6)\}$ and $J_{1}=\{1\}, J_{2}=\{2,3\}$.

We calculate $\mathcal{E}_{\infty}(f)$. Take $J \in J_{1} \times J_{2}$. Then $J=\{1,2\}$ or $J=\{1,3\}$. We calculate

$$
\alpha_{\{1,2\}}^{\max }=\max \left\{|\alpha|: \alpha \in V \cap \mathbb{R}^{\{1,2\}}\right\}=\max \{|\alpha|: \alpha \in\{(8,4)\}\}=12
$$

and

$$
\alpha_{\{1,3\}}^{\max }=\max \left\{|\alpha|: \alpha \in V \cap \mathbb{R}^{\{1,3\}}\right\}=\max \{|\alpha|: \alpha \in\{(8,6)\}\}=14 .
$$

Finally

$$
\mathcal{E}_{\infty}(f)=\min \left\{\alpha_{\{1,2\}}^{\max }, \alpha_{\{1,3\}}^{\max }\right\}=\min \{12,14\}=12
$$

Open Access This article is distributed under the terms of the Creative Commons Attribution 4.0 International License (http://creativecommons.org/licenses/by/4.0/), which permits unrestricted use, distribution, and reproduction in any medium, provided you give appropriate credit to the original author(s) and the source, provide a link to the Creative Commons license, and indicate if changes were made.

\section{References}

Bùi, N.T.N., Pham, T.S.: Computation of the Łojasiewicz exponent of nonnegative and nondegenerate analytic functions. Int. J. Math. 25, 1450092 (2014)

Đinh, S.T., Hà, H.V., Tháo, N.T.: Łojasiewicz inequality for polynomial functions on non-compact domains. Int. J. Math. 23, 1250033 (2012) 
Đinh, S.T., Kurdyka, K., Le Gal, O.: Łojasiewicz inequality on non-compact domains and singularities at infinity. Int. J. Math. 24, 1350079 (2013)

Đinh, S.T., Hà, H.V., Pham, T.S., Tháo, N.T.: Global Łojasiewicz-type inequality for non-degenerate polynomial maps. J. Math. Anal. Appl. 410, 541-560 (2014)

Gindikin, S.G.: Energy estimates connected with the Newton polyhedron. (Russian) Collection of articles dedicated to the memory of Ivan Georgievič Petrovskiı̌. Trudy Moskov. Mat. Obšč. 31, 189-236 (1974)

Gwoździewicz, J.: Growth at infinity of a polynomial with a compact zero set. Banach Cent. Publ. 44, 123-128 (1998)

Hà, H.V., Duc, N.H.: Łojasiewicz inequality at infinity for polynomials in two real variables. Math. Z. 266, 243-264 (2010)

Hà, H.V., Ngai, H.V., Pham, T.S.: A global smooth version of the classical Łojasiewicz inequality. J. Math. Anal. Appl. 421, 1559-1572 (2015)

Hörmander, L.: On the division of distributions by polynomials. Ark. Mat. 3, 555-568 (1958)

Kollár, J.: Sharp effective Nullstellensatz. J. Am. Math. Soc. 1, 963-975 (1988)

Łojasiewicz, S.: Sur le problème de la division. (French). Stud. Math. 18, 87-136 (1959) 